\title{
Work Integrated Learning (WIL): Integrating Frameworks for Education and Practice
}

\section{Sarah Jeong}

Ph.D., RN MN Grad Dip (Aged care) BN

Associate Professor, School of Nursing and Midwifery

University of Newcastle, Australia

\author{
Margaret McMillan \\ OAM Ph.D., RN BA MCurr St (Honours) DNE \\ Emeritus Professor, School of Nursing and Midwifery \\ University of Newcastle, Australia
}

\begin{abstract}
Objectives: To explore i) the nature and extent to which work oriented placements at an international level offer opportunities to experience actual and contemporary practice and ii) gain new insights into the complexities of applying concepts acquired in virtual learning situations and to determine the extent to which assessment of learning outcomes focuses on elements of: acquisition of abilities, requirements for professional competence and confidence, the honing of skills sets.
\end{abstract}

Methods: A mixed methods approach was used including; a focus group interview, literature review; identification of pedagogy and approaches used in field experiences; identification of best practices with respect to learning outcomes. Participants include all stakeholders: Curriculum developers, education providers, students, consumers of health services.

Results: There is a continuum representing a spectrum of WIL activities conducted both locally in Australia and internationally and there are four broad categories of evidence of outcomes of WIL irrespective of the context of practice; 1) Reflections on real work/real time activities, including direct observation and third party reports, 2) The need for use of structured activities in simulation, demonstration and activity sheets; 3) Questioning, including oral and written (including international work-related projects); and 4) Keeping records in portfolios and/or actual current workplace documentation of activities.

Conclusions: Further research needs to be undertaken to develop guiding principles to assess the extent to which assessment tasks in WIL at an international level are: Valid, sufficient, current and authentic with respect to the needs of the profession and the people for whom they are caring.

Development of guidelines and frameworks that include international professional placements has the potential to 1) optimise learning outcomes and experiences of students at Undergraduate and Graduate levels, 2) provide educators with principles, best practices guidelines to check the validity of assessment tools and learning resources, and 3) inform policy, procedure and practices for all stakeholders: teachers, students and consumers of health care.

Keywords: Cultural competence, Work integrated learning, assessment for competence and confidence, curriculum development 


\section{BACKGROUND}

Work Integrated Learning (WIL), 'in context' (locally and within national or international contexts) is meant to extend the university acquired knowledge and build the capacities required to engage in and be an effective professional practitioner (Billett, 2011). It is a form of academic 'service learning' offered through a formal academic curriculum that is rooted in the discipline in which the course is offered. The learning activities are organized through a set of practice-based learning events through which students directly address identified learning needs but may also provide a service to the community and determine other learning needs which arise from their exposure to actual practice. The real context of practice offers structured opportunities for students to connect their service activities to the course curriculum through novel situations arising from the dynamic environment in which professionals work.

The workplace is becoming more and more important as a source of contemporary knowledge and skill acquisition because of the rapidly changing nature of health services globally. Health professional education should have as its primary goal to assist students to become health professionals to work in a range of contexts of practice. The University of Newcastle in Australia, through its tradition of Problem-Based Learning (PBL) for the professions and its emphasis on community and industry partnerships across all areas of teaching and learning and research has established a long and active track record of approaches to experiential learning, locally and internationally. These, in broad terms are elements of 'integrated learning' including access to learning in the workplace and are understood to reflect the principles of 'engaged scholarship'. There is a recognition of the relationship between experiential learning and the necessary post-graduate experience and values such as 'socially responsible citizenship' as expressed within the 'Graduate Attributes' that inform learning outcomes through assessments and the need for consistency between discipline-specific scholarship and practice. Of particular interest to international placement experiences is students' ability to accommodate cultural diversity, to demonstrate sensitivity and cross-cultural awareness and to see the salient aspects of therapeutic interventions in a novel context of practice.

Australia is a multicultural country. In 2009, at least 46 per cent of Australian population was born overseas or had a parent born overseas (ABS 2012). This means that inevitably students in health care disciplines have clinical encounters that are multicultural in nature. Understanding and sensitivity to the languages, cultural beliefs and values of Australia's many cultural, cultural groups is an important feature of university experience but health science students' needs are more profound. They need to build on this diversity to work effectively with people from other cultures. While Billett's (2011) research on professional placement activity did not extend to international activities that involve professional placements, there is a growing body of literature on the benefits of international clinical placements. To date however, few studies have explored this phenomenon comprehensively and the fundamental questions that focus on the value of such activities remain unanswered.

\section{LITERATURE REVIEW}

Billett (2011) developed the 'Guidelines for practice: integrating practice-based experiences' based around six participating universities' experiences of teaching courses that included practice-based components. He clearly noted that simply providing clinical experiences for students 
does not guarantee the kinds of learning required to secure smooth transitions to practice and the development of effective and critically-orientated professional practitioners. He emphasised that students as active learners need to be engaged, prepared and extended in order for them to be able to integrate those experiences across practice and higher education settings and to engage in professional practice with critical and reflexive mindsets. However, the current practice and extent of short-term study abroad programs for students in Australian universities are not known. How the pedagogical approaches work and under what conditions educational benefits are embedded in those programs also needs to be scrutinised in order to enhance learning outcomes and experiences related to cultural competence and safety.

Universities are adopting an increasingly international focus to encourage students to engage with the global community. Students themselves travel and expect curricula that are international in scope and focus. At a policy level, the New Colombo Plan developed by the Australian government stresses the important role of universities in developing their graduates' global perspectives that foster cultural competence and intercultural understanding, especially in the Asian region. Consequently, there has been a growing recognition of the need for medical, nursing and allied health students to gain the knowledge, skills and personal experiences to prepare them to work effectively in an increasingly globalized and culturally diverse workplace. To meet this need, students are being offered short term international clinical placement opportunities as part of their education and training. International placement programs have been developed for doctors (Niemantsverdriet et al., 2006), nurses (Grant \& McKenna, 2003; Koskinen \& Jokinen 2007; Morgan, 2012; Tuckett \& Crompton, 2012), occupational therapists, physiotherapists, and speech language therapists (Peyinh et al., 2012; Pickering \& McAllister 1999).

Australian Government policy is also evident in the initiatives supported by the Office for Learning and Teaching (OLT: formerly Australian Learning and Teaching Council: ALTC). The OLT has sponsored a number of projects that are relevant to the topic of developing global perspectives. Leask and Wallace (2011) identified in the 'Good Practice Report: Learning and Teaching Across Cultures 2012' three key areas that were supported by the ALTC. These include; 1) the development of intercultural competence, 2) student support, and 3) transnational teaching and learning. These projects include the following foci of relevance:

- Attempts to develop intercultural competence by 1) developing a framework and a wide range of resources disseminated through a series of seminars (CG6-37), 2) internationalization of the curriculum (National Teaching Fellowship 2010), 3) addressing major issues faced by international students such as English language entry requirements, pre-departure preparation, orientation and transition to studentcentred learning (CG7-494), and 4) developing practical resources to enhance the experience of Australian students in study abroad (CG10-1549).

- Projects relevant to student support including; 1) institutional strategies to develop English language skills among international students, 2) important factors that particularly influence quality of learning for international students, 3) student preferences in support and reference materials in arts programs, and 4) resources suitable to the learning needs of Chinese and refugee students entering a world of studentcentred, independent, and critical learning. 
- Projects relevant to translational learning and teaching; 1) identifying the challenges of consistency in assessment in 'on and offshore' locations in accounting, 2) developing a framework to address curriculum, pedagogy and welfare issues in offshore programs, and 3) developing a toolkit for use in assessment moderation.

Another project which was funded more recently by OLT in 2013 is 'Internationalisation at home: Enhancing intercultural capacities of business and Health teachers, students and curricula was conducted by Mak et al. (2013). The focus of this project was on capacity-building through institutional and national presentations and showcases, online dissemination of intercultural curriculum resources, and scholarly reports of the impact of curriculum changes on student outcomes. This is the only project funded by the OLT which included the health disciplines (nursing, pharmacology and psychology).

Another gap identified in the current literature is that 'student mobility' which is one of the five key areas relevant to developing global perspectives. Of the 13 studies funded by the OLT, 'Bringing the learning home: Programs to enhance study abroad outcomes in Australian universities' (Gothard, Gowney, \& Gray, 2012) is the only project that is directly relevant to the project proposed and that has its focus on outbound students. Although their project aimed to facilitate a sector-wide improvement in approaches to the study abroad experience, the focus of their project centres on 1) developing teaching resources to support academic staff in internationalisation of higher education curricula, and 2) learning and teaching materials for Australian students which can be adapted for presentation on all Australian campuses. The fundamental questions of what ensures a successful international clinical placement and how we assess students of the outcomes remain unanswered. Given the financial costs and efforts involved in short term mobility programs at individual, universities and government levels, finding the answers to these questions is warranted.

\section{AIMS}

The aims of Jeong's (2014) research were;

1) to explore the nature and extent to which workoriented placements at an international level offer the opportunity to experience actual and contemporary practice in context and gain new insights into the complexities of applying the concepts and principles of problem or practice-based experiences in acquired virtual learning situations, and

2) to determine the extent to which assessment of learning outcomes focuses on elements of:

- acquisition of abilities,

- requirements for professional competence and confidence,

- the honing of skill sets.

\section{METHODS}

A mixed methods approach was used including; qualitative explorative focus group meetings with the students who participated in a short term international placement; literature review; identification of pedagogy and approaches used in field experiences; and identification of best practices with respect to learning outcomes. Participants include all stakeholders: Curriculum developers, education providers, students, and consumers of health services. 


\section{RESULTS}

Students undertaking this extra-curricular activity were nevertheless using their own undergraduate studies as their learning platform. Appraisal of their curriculum model which focuses on transformative learning (Mezirow, 1978, King, 2002), demonstrated that it is characterized by elements consistent with problem or practice-based curricula and WIL:

- the use of stimulus material that reflects the dilemmas that represent nursing issues and contexts for learning

- a focus on higher order thinking skills
- aspirations for integration of knowledge, skills and behaviours

- learning in groups and working as a team member

- self-directed learning, particularly through a range of media including on-line.

There is a continuum representing a spectrum of WIL activities conducted both locally in Australia and internationally and there are four broad categories of evidence of outcomes of WIL irrespective of the context of practice; 1) reflections on real work/real time activities, including direct observation and third party reports, 2)

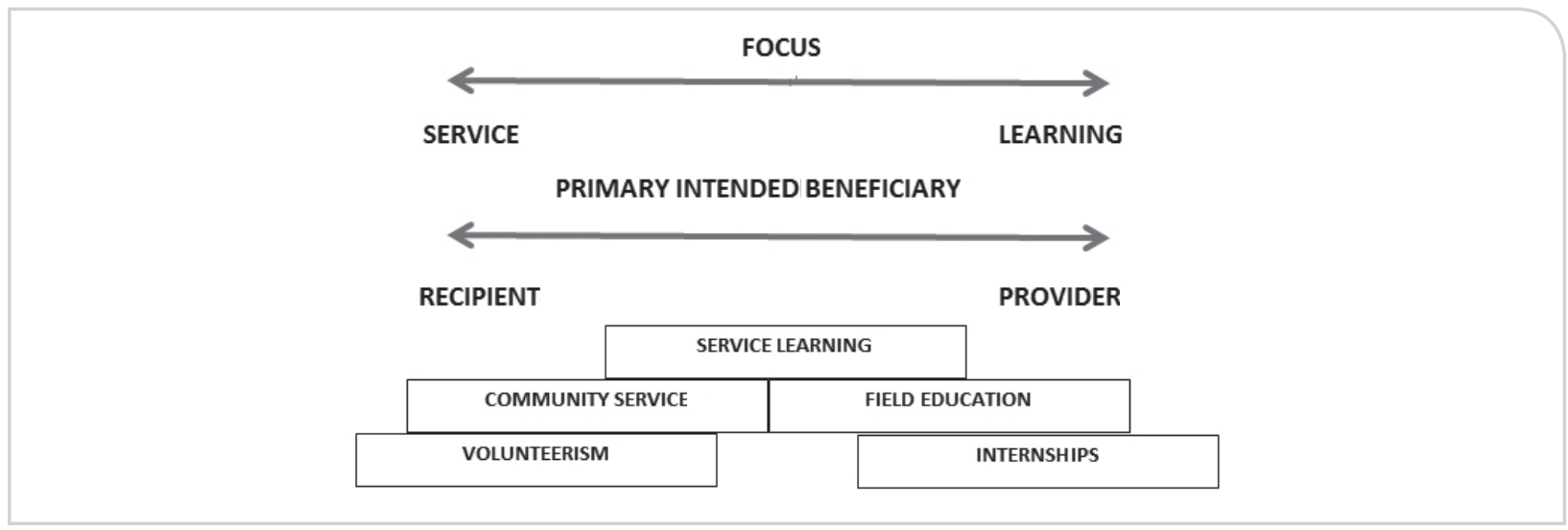

Figure 1. Professional placement learning continuum

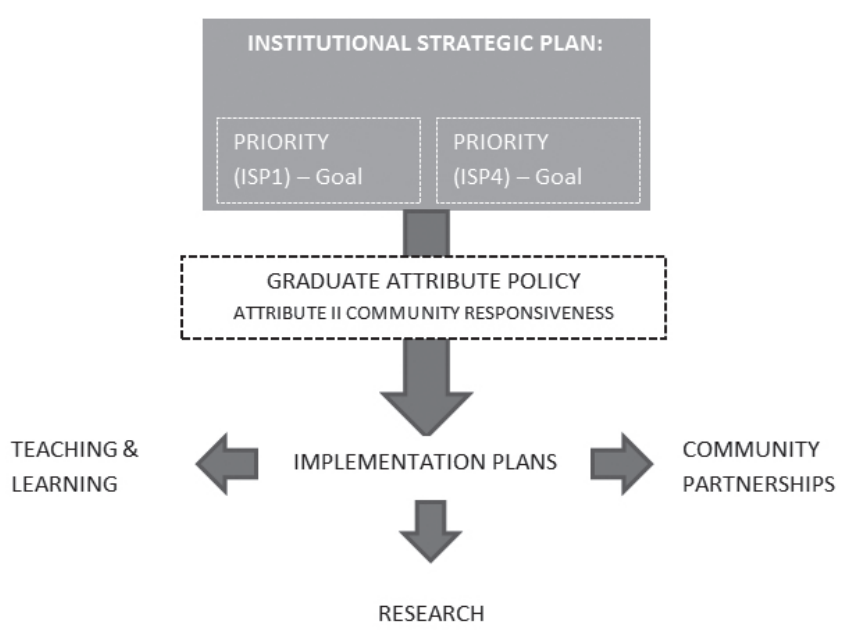

Figure 2. Strategic framework for supporting engaged scholarship at the University 
the need for use of structured activities in simulation, demonstration and activity sheets; 3 ) questioning, including oral and written (including international workrelated projects); and 4) keeping records in portfolios and/ or actual current workplace documentation of activities.

Further exploration of the implementation processes used within the international experience included interrogation of the extent to which students were given the opportunity to evaluate what they observed on their international experience and suggest alternatives to what they see in actual practice. There was insufficient opportunity for students to think about what needs to be done for the consumer of care or experiences which enhanced the likelihood of graduates assuming an appropriate professional identity. Learning events need to provide more opportunities to explore relationships among the theory, appropriate decision-making and professional judgment and actions. There was not enough opportunity for development of the skill inherent within enquiry processes (the thinking about) that enables students to develop knowledge about their professional practice within the complex reality of the workplace. This is about the students acquiring 'Knowledge for action'. In the acquisition of discipline specific knowledge (content) needed to be processed and the 'results in action' tested for appropriateness to that discipline in actual practice. Students needed to have the opportunity to develop general principles of managing practice; which are portable across contexts. There was a need to assess whether or not they were able to integrate concepts that have learned to those that they see in the real world of practice in other contexts and cultures.

These desirable learning outcomes outlined above are consistent with Practice-based learning representative

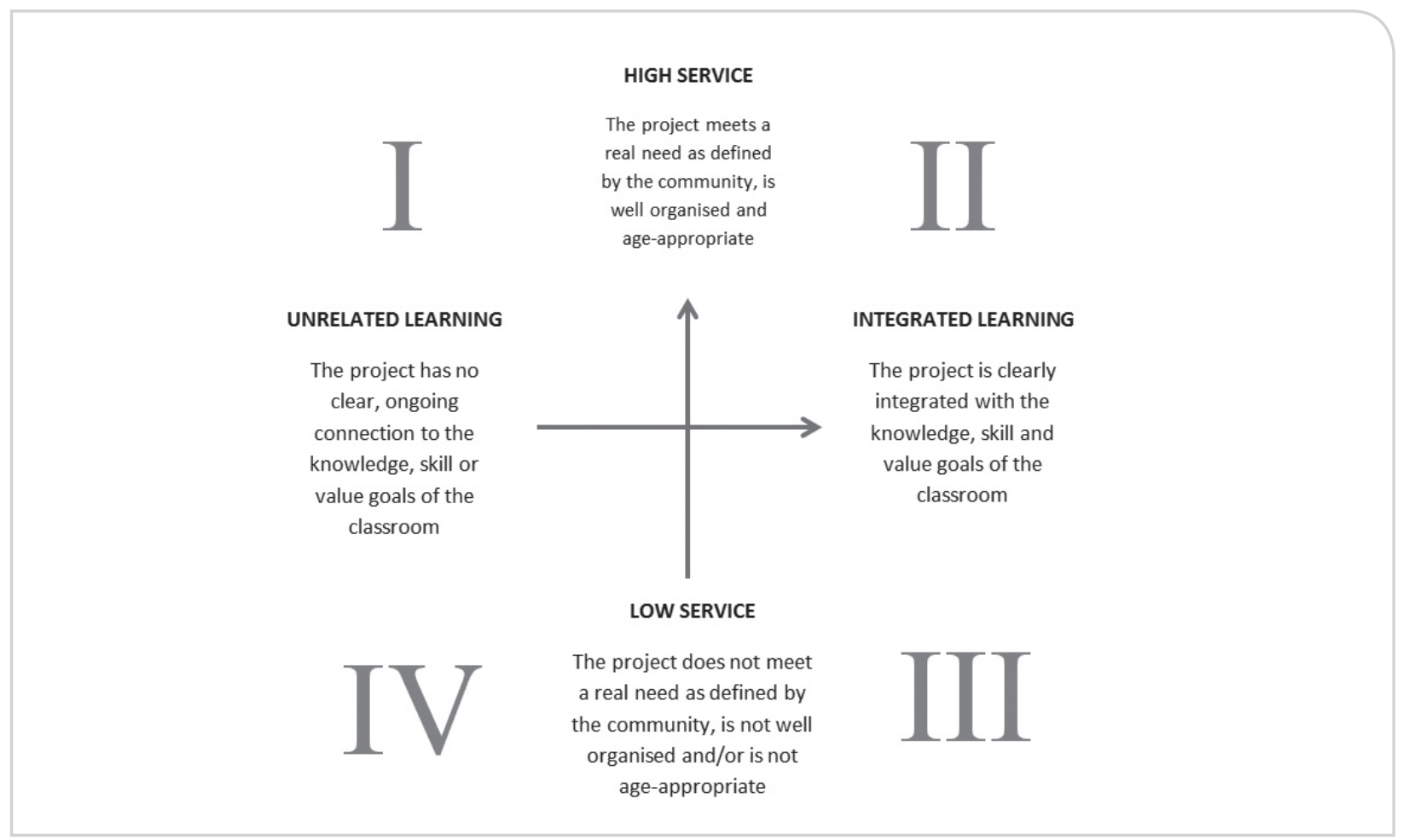

Figure 3. Evaluating integrated and service learning in an international context of practice 
of WIL. Figures 1, 2 and 3 present the key concepts and various levels of student engagement within a professional placement in an international context of practice.

One could see from the Australian study conducted by Jeong (2014), that student participants reported that an extra-curricular 3-week community placement in Cambodia provided them with an opportunity to experience health care services and systems in another culture and to share their knowledge and skills with people of another country who have poor living quality of life and social status. Similarly Stone et al. (2014) found that students had undergone profound learning on a two weeks study tour of Northern Thailand. However, these WIL experiences would be better conceptualized within a learning framework and be part of the formal curriculum. Given that a well constructed learning event is essential for students to be able to integrate those international experiences into practice, the absence of a framework and guidelines to design and implement educationally sound learning event through short-term abroad programs imposed a great challenge for students, academics and the people with whom they were working. The students also encountered great challenges when they were confronted with poverty, language barriers, and feelings of not being able to help enough. There needs to be careful management of these responses as part of their reflection on the experience.

\section{DISCUSSION}

Careful application of the elements of WIL which are best developed in workplaces because this offers the best opportunity to experience actual and contemporary practice; those practices reflective of what professionals actually do. These can be local, more remote from the home institution or even in countries foreign to the students' prior experience. WIL centres on student learning about an occupation and the variations within different contexts and practice settings in which professional and vocational groups work. Professional experience placements provide the opportunity for students to observe and participate in practice and reflect on the effectiveness of the professional's practice. They can assess their own ability to make and reflect on the soundness of clinical judgments based on assessments and reassessments, to collaborate with others from within their own discipline or with others, to provide meaningful feedback to colleagues about performance, and to establish and maintain professional relationships. Clinical experiences acclimatise students to the real world of practice and its culture, providing preparation for the reality of practice which is dynamic and replete with novel situations and opportunities to bring these new insights back to test conceptual frameworks and theories informing practice.

Nevertheless the benefits of overseas education experiences to students are well documented (Malicki \& Potts, 2013) and include increased: 1) international skills, 2) academic results, 3) personal awareness and an international outlook, and 4) intercultural awareness. The absence of a framework diminishes the extent to which students can embellish their thinking about the practice with which they want to engage. A framework provides the pedagogical context and underpinning values and evidence base for practice. How we think about a practice shapes what we learn from practice.

Another challenge noted within the international experiences, as well as the local placements, is that there are tensions in working with actual practice between what practice is and what practice should be and the practice 
exemplars developed by educators for student learning experiences often do not reflect the reality of practice in some context. There was evidence of this in the students' responses in Jeong's study (2014).

Whilst ways of securing instances of authentic practice are recommended by Billett (2011), this is not only challenging for students but also for educators. The key considerations for organising students' learning experiences (Billett, 2011) provide educators with some direction. For example, 'students' interest in and readiness for the learning activity is central to their engagement and learning in practice settings, and reconciling it with what they have learnt in their courses'. 'Teachers' knowledge of and engagement with practice settings influence how they enact experiences for their students. This is critical in situations involving international placement experiences.

\section{CONCLUSION}

A framework for short term international clinical placement based on a more extensive piece of research is needed to draw on the latest national and international research and good practice in learning and teaching. The outcomes of the project includes a proposal for development of a framework that integrates the three most relevant works by; 1) Billett (Curriculum and pedagogic bases for effectively integrating practice-based experiences, 2011), 2) Leask (Internationalisation of the Curriculum in Action, 2012), and 3) Gothard and her colleagues (Bringing the learning home: Programs to enhance study abroad outcomes in Australian universities, 2012). Any framework needs to be based on additional research but must have the potential to link internationalisation of curriculum and pedagogic principles. It is important to consider ways of achieving the integration of concepts presented to students before, during and after short term international clinical placements, and the requirements of professional practice and citizenship in institutional, local, national and global contexts.

However the project allowed for a comprehensive approach to ensuring that international clinical placements not only enhance students' learning experience in overseas countries but also enhance culturally appropriate professional behaviours in their home country. Language barriers can be a problem for some students; this was the case for business students on a tour of China despite careful pre- trip preparation (Hutchings, Jackson, \& McEllister, 2002)

This project centred on one international initiative which has the potential to address the aspirations for 'student mobility' related to experience across cultures, one of the Australian Government's priorities. Given the political and financial commitment by the Australian government, anecdotal evidence suggests that more and more universities are adopting 'study abroad' programs.

The project explored the potential for expanding on an important issue in higher education, that of 'developing global perspectives' by university students as a pathway to employability in Australia and worldwide. This is an avenue for sustainable people-to-people and institutional relationships with national and international partners. While various forms of volunteering opportunities are also available to students, this project focussed specifically on identifying the current practice and extent of 'shortterm study abroad' programs for nursing students to establish fundamental information for all universities and to improve students' learning experiences and educators' teaching experiences. Educational principles will however need to be identified and translated into the WorkIntegrated Learning (WIL) in order to enhance learning 
and to produce a good practice guide.

Additional research needs to be undertaken. The use of a strategic approach is necessary to encompass four levels (principles, policies, guidelines, and procedures) by involving all stakeholders (students, educators, and organisations offering study abroad programs) to capture the full picture of short-term international clinical placement for nursing and health professional disciplines.

There is a need to deliver to Australian universities, national guidelines that are transferable to a range of disciplines to assist students and educators to effectively prepare and undertake short term international clinical placements. The appraisal of local experiences of international placements highlighted the nature and extent of return on investment (ROI) to the student and the School, and provided the researchers with essential information to ensure the outbound short term options for WIL were consistent with curriculum objectives.

The project identified the need to undertake further research to develop a set of national guidelines for Australian higher education providers and organisations that offer short term health oriented clinical placements as part of the study abroad programs to ensure program design, assessment, and student experiences are of high quality. Evidence of quality in learning outcomes as a result of teaching approaches is necessary. The development of a comprehensive and specific set of guidelines promotes and supports change within higher education institutions for enhancement of learning and teaching, and the benefit of the students' experiences in short term international clinical placement in an area of strategic priority, related to issues around expanding cultural competence of students and enhancing aspects of globalisation.

\section{REFERENCES}

Australian Bureau Statistics (ABS) (2012) Cultural diversity in Australia: Reflecting a Nation: Stories from the 2011 census. Available at http://www.abs. gov.au/ausstats/abs@.nsf/lookup/2071.0main+featur es902012-2013 (accessed 13 February 2014).

Billett, S. (2011). Curriculum and pedagogic bases for effectively integrating practice-based experiences. Sydney: Australian Learning and Teaching Council.

Gothard, J., Gray, T., Downey, G. (2012). Bringing the learning home: Programs to enhance study abroad outcomes in Australian Universities. Sydney: Australian Government Office for Learning and Teaching.

Grant, E., \& McKenna, L. (2003). International clinical placements for undergraduate students. Journal of Clinical Nursing, 12, 529-535.

Hutchings, K., Jackson, P., \& McEllister, R. (2002). Exploiting the Links between

Theory and Practice: Developing Students' Crosscultural Understanding through an International Study Tour to China. Higher Education Research \& Development, 21(1), 55-71. doi: 10.1080/07294360220124657

Jeong, S. (2014). Community health placement in Cambodia: Pre and post evaluation. University of Newcastle. Australia

Koskinen, L., Jokinen, P. (2007). Multicultural healthcare: A transatlantic project. Nurse Educator, 32, 89-93.

Leask, B. (2012). Internationalisation of the curriculum in action. Sydney: Australian Government Office for Learning and Teaching.

Leask, B., \& Wallace, J. (2011). Good practice report: Learning and teaching across cultures. Sydney: Australian Learning and Teaching Council.

Mak, A., Grealish, L., Daly, A., \& Neill, J. (2013). Internationalisation at home: Enhancing intercultural capabilities of business and health teachers, students and curricula. Sydney: Australian Government Office for Learning and Teaching. 
Malicki, R., \& Potts, D. (2013). The outcomes of outbound student mobility: A summary of academic literature. AIM Overseas.

King, K. P. (2002). A journey of transformation: A model of educators' learning experiences in educational technology. In J. M. Pettit \& R. P. Francis (Eds.). Proceedings of the 43rd Annual Adult Education Research Conference, (pp. 195-200). Available: http:// www.adulterc.org/Proceedings/2002/papers/King. pdf

Mezirow, J. (1978). Perspective transformation. Adult Education Quarterly, 28(2), 100-110.

Morgan, D. (2012). Student nurse perceptions of risk in relation to international placements: A phenomenological research study. Nurse Education Today, 32, 956-960.

Niemantsverdriet, S., Majoor GD., van der Vleuten CPM., Scherpbier, AJJA. (2006). Internationalization of medical education in the Netherlands: State of affairs. Med. Teac, 28, 187-189.

Nilson, C. (2011). International student nurse clinical placement: a supervisor's perspective Australian Nursing Journal, 19(3): 35.

Pickering, M., McAllister, L. (1999). Cross-cultural interaction: An essential component in speechlanguage pathology clinical education and professional development, in P. Dejonckere \& H.F.M Peters (Eds), Communication and its disorders: $A$ science in progress, pp. 946-949. Amsterdam, The Netherlands.

Peiying, N., Goddard, T., Gribble, N., Pickard, C. (2012). International placements increase the cultural sensitivity and competency of professional health students: A quantitative and qualitative study, Journal of Physical Therapy Education, 26(1), 61-68.

Stone, N. (2009). 'The curse of the constructs: defining and measuring intercultural effectiveness', paper delivered to the 23rd Annual Australian New Zealand Academy of Management Conference.

Stone, T. E., Francis, L., van der Riet, P., Dedkhard, S., Junlapeeya, P., \& Orwat, E. (2014). Awakening to the other: Reflections on developing intercultural competence through an undergraduate study tour. Nurs Health Sci, 16(4), 521-527. doi: 10.1111/nhs.12139

Sutton, RC. \& Rubin, DL. (2004). 'The GLOSSARI project: initial findings from a system-wide research initiative on study abroad learning outcomes', Frontiers: The interdisciplinary Journal of Study Aboard, 10, 65-82.

Tuckett, A., \& Crompton, P. (2013). Qualitative understanding of an international learning experience: What Australian undergraduate nurses and midwives said about a Cambodia placement? International Journal of Nursing Practice. Doi: 10.1111/ijnp.12142.

University of Newcastle. (2008). Report on the 'Scoping Study of 'Service Learning' opportunities within undergraduate Programs at the University of Newcastle, University of Newcastle, Australia 